J. KSIAM Vol.16, No.2, 85-91, 2012

\title{
ENERGY DECAY RATES FOR THE KELVIN-VOIGT TYPE WAVE EQUATION WITH ACOUSTIC BOUNDARY
}

\author{
YOUNG IL SEO ${ }^{1}$ AND YONG HAN $\mathrm{KANG}^{2, \dagger}$
}

\footnotetext{
${ }^{1}$ National Fisheries Research and Development Institute, Busan 619-705, South Korea

E-mail address: seovi@nfrdi.go.kr

${ }^{2}$ Institute of Liberal Education, Catholic University of Daegu, Gyeongbuk 712-702, South Korea

E-mail address: yonghannecu.ac.kr
}

\begin{abstract}
In this paper, we study uniform exponential stabilization of the vibrations of the Kelvin-Voigt type wave equation with acoustic boundary in a bounded domain in $R^{n}$. To stabilize the systems, we incorporate separately, the internal material damping in the model as like Gannesh C. Gorain [1]. Energy decay rates are obtained by the exponential stability of solutions by using multiplier technique.
\end{abstract}

\section{INTRODUCTION}

In this paper, we consider the uniform stability of a mathematical problems governed by the following a nonlinear wave equations of the Kelvin-Voigt type with acoustic boundary conditions:

$$
\begin{aligned}
& u^{\prime \prime}=\left(a^{2}+b \int_{\Omega}|\nabla u|^{2} d x\right) \triangle u+2 \lambda \triangle u^{\prime} \text { in } \Omega \times R^{+}, \\
& u=0 \text { on } \Gamma_{0} \times R^{+}, \\
& \left(a^{2}+b \int_{\Omega}|\nabla u|^{2} d x\right) \frac{\partial u}{\partial \nu}+2 \lambda \frac{\partial u^{\prime}}{\partial \nu}=y^{\prime} \text { on } \Gamma_{1} \times R^{+}, \\
& u^{\prime}+p(x) y^{\prime}+q(x) y=0 \text { on } \Gamma_{1} \times R^{+}, \\
& u(0)=u_{0}, u^{\prime}(0)=u_{1} \text { in } \Omega,
\end{aligned}
$$

where $\Omega$ is a bounded, connected set in $R^{n}(n \geq 1)$ having a smooth boundary $\Gamma=\partial \Omega$, consisting of two parts $\Gamma_{0}$ and $\Gamma_{1}$ such that $\overline{\Gamma_{0}} \cup \overline{\Gamma_{1}}=\Gamma$. Primes denote the time derivative, $\Delta$ the Laplacian in $R^{n}$ taken in space variables, $\nu$ the unit normal of $\Gamma$ pointing towards exterior of $\Omega$ and $R^{+}:=(0, \infty)$. The parameters $\lambda>0$ is a small internal material damping coefficient, and $a>0, b>0$ are constant real

Received by the editors February 11 2012; Revised June 7 2012; Accepted in revised form June 152012.

2010 Mathematics Subject Classification. 35L70, 35B40, 76Exx.

Key words and phrases. Kelvin-Voigt type, Energy decay, Acoustic boundary, Stabilization, Lyapunov functional.

${ }^{\dagger}$ Corresponding author.

This study was founded by the National Fisheries Research and Development Institute (RP-2012-FR-024). 
numbers. $p$ and $q$ are functions satisfying some conditions to be specified later. Physically, the integrodifferential equations (1.1)-(1.5) occurs in the study of vibrations of damped flexible space structures in bounded domain in $R^{n}$. The term $2 \lambda \Delta u^{\prime}$ is the internal material damping of Kelvin-Voigt type of the structure. The boundary conditions considered here are of mixed Dirichlet and Neumann type and acoustic boundary. The analytical studies in the area of stabilization of distributed parameter system is currently of interest in view of application to vibration control of various structural elements. The phenomenon was first observed by Hunton as reported by Harrison [7]. The nonlinear model like (1.1) for transverse vibrations was originally derived by Kirchhoff [3]. Beale and Rosencrans[5] introduced acoustic boundary conditions of the general form

$$
\begin{aligned}
& \frac{\partial u}{\partial \nu}=y^{\prime} \text { on } \Gamma_{1} \times R^{+} \\
& \gamma u^{\prime}+m(x) y^{\prime \prime}+p(x) y^{\prime}+q(x) y=0 \text { on } \Gamma_{1} \times R^{+} .
\end{aligned}
$$

Recently, wave equations with acoustic boundary conditions have been treated by many authors $[4,5,6$, $8,9,11,12,13]$. In [4], the authors studied the nonlinear wave equations

$$
\begin{aligned}
& u^{\prime \prime}-M\left(\int_{\Omega}|u|^{2} d x\right) \Delta u+\left|u^{\prime}\right|^{\alpha} u^{\prime}=0 \text { in } \Omega \times R^{+}, \\
& u=0 \text { on } \Gamma_{0} \times R^{+}, \\
& \frac{\partial u}{\partial \nu}=y^{\prime} \text { on } \Gamma_{1} \times R^{+}, \\
& \gamma u^{\prime}+m(x) y^{\prime \prime}+p(x) y^{\prime}+q(x) y=0 \text { on } \Gamma_{1} \times R^{+} .
\end{aligned}
$$

They proved the existence of solutions, but did not give decay rate for solutions. As regards uniform decay rates for solutions to problems with acoustic boundary conditions, there are not much literature [2],[4],[9],[11],[12]. Frota and Larkin[9] established global solvability and decay estimates for a linear wave equation with boundary conditions

$$
\begin{aligned}
& \frac{\partial u}{\partial \nu}=h(x) y^{\prime} \text { on } \Gamma_{1} \times R^{+} \\
& \gamma u^{\prime}+p(x) y^{\prime}+q(x) y=0 \text { on } \Gamma_{1} \times R^{+} .
\end{aligned}
$$

In this paper we are motivated by boundary conditions of Park[4] and results of Gorain[1] and Kang[10]. The aim of this paper is to study uniform stabilization of the generalized nonlinear Kirchhoff type wave equations governed by (1.1)-(1.5) with the mixed boundary conditions. To our knowledge, this problem has not been considered by predecessors and is studied first, as a Kirchhoff of kelvin-Voigt type model, in this paper. The plan of this paper as follows. In section 2, we give some notation, some conditions and 
material needed for our work. In section 3, we drive the uniform stability on account of internal material damping of Kelvin-Voigt type with acoustic boundary. The notation used in this paper is standard and can be found in Gorain[1].

\section{PRELIMINARIES AND SOME NOTATIONS}

In this section, we present some notations and some material in the proof of our result. Throughout this paper, we use the notation $V=\left\{u \in H^{1}(\Omega): u=0\right.$ on $\left.\Gamma_{0}\right\}$ the subspace of the classical Sobolev space $H^{1}(\Omega)$ of real valued functions of order one. Let $k$ be the smallest positive constant independent of $t$ (depends only on $\Omega$ ) satisfying the Poincare inequality

$$
\int_{\Omega} u^{2} d x \leq k \int_{\Omega}|\nabla u|^{2} d x \text { for every } u \in V .
$$

And also let $\bar{k}$ be the smallest positive constant independent of $t$ (depends only on $\Gamma_{1}$ ) satisfying the embedding inequality

$$
\int_{\Gamma_{1}} u^{2} d \Gamma \leq \bar{k} \int_{\Omega}|\nabla u|^{2} d x \text { for every } u \in V
$$

For the functions $p$ and $q$, we assume that $p, q \in C\left(\Gamma_{1}\right)$ and $p(x)>0$ and $q(x)>0$ for all $x \in \Gamma_{1}$. This assumption implies that there exist positive constants $p_{i}, q_{i}(i=0,1)$ such that

$$
p_{0} \leq p(x) \leq p_{1}, q_{0} \leq q(x) \leq q_{1} \text { for all } x \in \Gamma_{1} .
$$

By using Gälerkin's approximation and the methods of Gorain[1] and Park[12], we can obtain the following existence result for the solution subject to (1.1)-(1.5) under the conditions on $p$ and $q$ as above. For the initial data $\left(u_{0}, u_{1}\right) \in\left(V \cap H^{2}(\Omega)\right) \times V$, there exists a unique pair of functions $(u, y)$, which is a solution to the problem (1.1)-(1.5) in the class

$$
\begin{aligned}
& u \in L^{\infty}\left(0, T ; V \times H^{2}(\Omega)\right), \quad u^{\prime} \in L^{\infty}(0, T ; V), \\
& u^{\prime \prime} \in L^{\infty}\left(0, T ; L^{2}(\Omega)\right), \quad y, y^{\prime} \in L^{2}\left(0, \infty ; L^{2}\left(\Gamma_{1}\right)\right) .
\end{aligned}
$$

In the order to state our main results, we define the energy of problem (1.1)-(1.5) by

$$
E(t)=\frac{1}{2} \int_{\Omega}\left(u^{\prime}\right)^{2} d x+\frac{a^{2}}{2} \int_{\Omega}|\nabla u|^{2} d x+\frac{b}{4}\left(\int_{\Omega}|\nabla u|^{2} d x\right)^{2}+\frac{1}{2} \int_{\Gamma_{1}} q(x)(y)^{2} d \Gamma .
$$


3. UNIFORM STABILITY ON ACCOUNT OF INTERNAL DAMPING OF KELVIN-VOIGT TYPE

If we differentiate (2.4) with respect to $t$ and use the governing Eq.(1.1) we get

$$
\begin{array}{r}
E^{\prime}(t)=\int_{\Omega}\left[u^{\prime}\left(a^{2}+b \int_{\Omega}|\nabla u|^{2}\right) \Delta u+2 \lambda u^{\prime} \Delta u^{\prime}+a^{2} \nabla u \cdot \nabla u^{\prime}\right] d x \\
+b \int_{\Omega}|\nabla u|^{2} d x \int_{\Omega} \nabla u \cdot \nabla u^{\prime} d x+\int_{\Gamma_{1}} q(x) y y^{\prime} d \Gamma .
\end{array}
$$

Application of Green's formula and using the boundary conditions (1.2)-(1.4) and then a simplification, we get

$$
E^{\prime}(t)=-2 \lambda \int_{\Omega}\left|\nabla u^{\prime}\right|^{2} d x-\int_{\Gamma_{1}} p(x)\left(y^{\prime}\right)^{2} d \Gamma \leq 0 \quad \forall t \in R^{+} .
$$

We see from (3.1) that the energy $E$ is a decreasing function of time and hence

$$
E(t) \leq E(0) \quad \forall t \geq 0
$$

where

$$
\begin{aligned}
E(0)= & \frac{1}{2} \int_{\Omega}\left[\left(u_{1}\right)^{2}+a^{2}\left|\nabla u_{0}\right|^{2}\right] d x+\frac{b}{4}\left(\int_{\Omega}\left|\nabla u_{0}\right|^{2} d x\right)^{2} \\
& +\frac{1}{2} \int_{\Gamma_{1}} q(x)(y(x, 0))^{2} d \Gamma .
\end{aligned}
$$

Under what conditions does this energy $E$ decay with time uniformly? An affirmative answer is contained in the following theorem.

Theorem 3.1. If $u=u(x, t)$ is a regular solution of the system (1.1)-(1.5) with initial values $\left(u_{0}, u_{1}\right) \in$ $V \times L^{2}(\Omega)$, then the energy $E(t)$ of the system defined by (2.4) satisfies

$$
E(t)<M e^{-\mu t} E(0), t \in R^{+}
$$

for some real constants $M>1(3.24)$ and $\mu>0(3.21)$, dependent on the damping parameter $\lambda(3.19)$.

Firstly, we need to prove the following lemma.

Lemma 3.1. For every solution $u=u(x, t)$ of the system (1.1)-(1.5), the time derivative of the functional G defined by

$$
\Psi(t)=\int_{\Omega}\left(u u^{\prime}+\lambda|\nabla u|^{2}\right) d x+\int_{\Gamma_{1}} u y d \Gamma+\frac{1}{2} \int_{\Gamma_{1}} p(x) y^{2} d \Gamma
$$

satisfies

$$
\Psi^{\prime}(t) \leq 2 k \int_{\Omega}\left|\nabla u^{\prime}\right|^{2} d x+2 \int_{\Gamma_{1}} u y^{\prime} d \Gamma-\frac{b}{2}\left(\int_{\Omega}|\nabla u|^{2} d x\right)^{2}-2 E(t), \forall t \in R^{+} .
$$


Proof. If we differentiate (3.3) with respect to $t$ and replace $u^{\prime \prime}$ by the relation (1.1), then we get

$$
\begin{aligned}
\Psi^{\prime}(t)= & \int_{\Omega}\left(u u^{\prime \prime}+\left(u^{\prime}\right)^{2}+2 \lambda \nabla u \cdot \nabla u^{\prime}\right) d x+\int_{\Gamma_{1}}\left(u^{\prime} y+u y^{\prime}\right) d \Gamma+\int_{\Gamma_{1}} p(x) y y^{\prime} d \Gamma \\
= & \int_{\Omega}\left[u\left(a^{2}+b \int_{\Omega}|\nabla u|^{2} d x\right) \triangle u d x+2 \lambda\left(u \triangle u^{\prime}+\nabla u \cdot \nabla u^{\prime}\right)\right] d x \\
& +\int_{\Omega}\left(u^{\prime}\right)^{2} d x+\int_{\Gamma_{1}} u y^{\prime} d \Gamma+\int_{\Gamma_{1}} y\left(u^{\prime}+p(x) y^{\prime}\right) d \Gamma .
\end{aligned}
$$

Applying Green's formula, we have

$$
\begin{aligned}
\Psi^{\prime}(t) & =\int_{\Omega}\left(u^{\prime}\right)^{2} d x-\left(a^{2}+b \int_{\Omega}|\nabla u|^{2} d x\right) \int_{\Omega}|\nabla u|^{2} d x \\
& +\int_{\Gamma} u\left[\left(a^{2}+b \int_{\Omega}|\nabla u|^{2} d x\right) \frac{\partial u}{\partial \nu}+2 \lambda \frac{\partial u^{\prime}}{\partial \nu}\right] d \Gamma+\int_{\Gamma_{1}} u y^{\prime} d \Gamma+\int_{\Gamma_{1}} y\left(u^{\prime}+p(x) y^{\prime}\right) d \Gamma
\end{aligned}
$$

Using the boundary conditions (1.2)-(1.4) and the energy (2.4), relation (3.6) can be written as

$$
\Psi^{\prime}(t)=2 \int_{\Omega}\left(u^{\prime}\right)^{2} d x+2 \int_{\Gamma_{1}} u y^{\prime} d \Gamma-\frac{b}{2}\left(\int_{\Omega}|\nabla u|^{2} d x\right)^{2}-2 E(t), \forall t \in R^{+}
$$

Hence the proof of lemma complete.

Proof of Theorem 1. We introduce a modified energy like Lyapunov functional $V$ by

$$
V(t)=E(t)+\frac{\lambda}{k} \Psi(t) \text { for } t \geq 0
$$

Now, using the Cauchy-Schwarz's inequality, the Poincare inequality(2.1)-(2.2) and the defined of energy (2.4), we obtain estimate as follow

$$
\begin{gathered}
\left|\int_{\Omega} u u^{\prime} d x\right| \leq \frac{\sqrt{k}}{2 a} \int_{\Omega}\left(\left(u^{\prime}\right)^{2}+\frac{a^{2}}{k} u^{2}\right) d x \\
\leq \frac{\sqrt{k}}{2 a}\left(\int_{\Omega}\left(u^{\prime}\right)^{2} d x+a^{2} \int_{\Omega}|\nabla u|^{2} d x\right) \leq \frac{\sqrt{k}}{a} E(t), \\
0 \leq \lambda \int_{\Omega}|\nabla u|^{2} d x \leq \frac{2 \lambda}{a^{2}} E(t), \\
\left|\int_{\Gamma_{1}} u y d \Gamma\right| \leq \int_{\Gamma_{1}} \frac{1}{2 q(x)} u^{2} d \Gamma+\frac{1}{2} \int_{\Gamma_{1}} q(x) y^{2} d \Gamma \\
\leq \frac{\bar{k}}{2 q_{0}} \int_{\Omega}|\nabla u|^{2} d x+\frac{1}{2} \int_{\Gamma_{1}} q(x) y^{2} d \Gamma \leq\left(\frac{\bar{k}}{a^{2} q_{0}}+1\right) E(t),
\end{gathered}
$$

and

$$
\frac{1}{2} \int_{\Gamma_{1}} p(x) y^{2} d \Gamma \leq \frac{p_{1}}{2 q_{0}} \int_{\Gamma_{1}} q(x) y^{2} d \Gamma \leq \frac{p_{1}}{q_{0}} E(t) .
$$


Thus the inequality (3.9)-(3.12) yield for $\Psi$ that estimates

$$
-\left(\frac{\sqrt{k}}{a}+\frac{\bar{k}}{a^{2} q_{0}}+1\right) E(t) \leq \Psi(t) \leq\left(\frac{\sqrt{k}}{a}+\frac{2 \lambda}{a^{2}}+\frac{\bar{k}}{a^{2} q_{0}}+\frac{p_{1}}{q_{0}}+1\right) E(t) .
$$

Then it follows from (3.13) that

$$
\begin{aligned}
\left(1-\frac{\lambda}{k}\left(\frac{\sqrt{k}}{a}+\frac{\bar{k}}{a^{2} q_{0}}+1\right)\right) E(t) \leq V(t) \leq \\
\left(1+\frac{\lambda}{k}\left(\frac{\sqrt{k}}{a}+\frac{2 \lambda}{a^{2}}+\frac{\bar{k}}{a^{2} q_{0}}+\frac{p_{1}}{q_{0}}+1\right)\right) E(t) \quad \forall t \geq 0,
\end{aligned}
$$

where we assume that

$$
0<\lambda<\frac{k}{\sqrt{k} / a+\bar{k} /\left(a^{2} q_{0}\right)+1}
$$

so that left hand side of (3.14) is positive.

Next, differentiating $V(t)$ ( defined by (3.8)) with respect to $t$ using expression $E^{\prime}(t)$ (defined by (3.1)) and Lemma 3.1, we have

$$
V^{\prime}(t)=-\int_{\Gamma_{1}} p(x)\left(y^{\prime}\right)^{2} d \Gamma-\frac{b \lambda}{2 k}\left(\int_{\Omega}|\nabla u|^{2} d x\right)^{2}+\frac{2 \lambda}{k} \int_{\Gamma_{1}} u y^{\prime} d \Gamma-\frac{2 \lambda}{k} E(t) .
$$

Now, using the Cauchy-Schwarz's inequality, the Poincare inequality, the conditions (2.2)-(2.3) and the definition of energy (2.4), we obtain estimate

$$
\begin{gathered}
\left|\frac{2 \lambda}{k} \int_{\Gamma_{1}} u y^{\prime} d \Gamma\right| \leq \int_{\Gamma_{1}} p(x)\left(y^{\prime}\right)^{2} d \Gamma+\frac{\lambda^{2}}{k^{2}} \int_{\Gamma_{1}} \frac{1}{p(x)} u^{2} d \Gamma \\
\leq \int_{\Gamma_{1}} p(x)\left(y^{\prime}\right)^{2} d \Gamma+\frac{2 \bar{k} \lambda^{2}}{a^{2} k^{2} p_{0}} E(t)
\end{gathered}
$$

From (3.16)-(3.17) and condition (3.15), we have

$$
\begin{aligned}
V^{\prime}(t) \leq- & \frac{b \lambda}{2 k}\left(\int_{\Omega}|\nabla u|^{2} d x\right)^{2}-\frac{2 \lambda}{k}\left(1-\frac{\bar{k} \lambda}{a^{2} k p_{0}}\right) E(t) \\
& <-\frac{2 \lambda}{k}\left(1-\frac{\bar{k} \lambda}{a^{2} k p_{0}}\right) E(t) \quad \forall t \in R^{+},
\end{aligned}
$$

where we assume that

$$
0<\lambda<\min \left\{\frac{a^{2} k p_{0}}{\bar{k}}, \frac{k}{\frac{\sqrt{k}}{a}+\frac{\bar{k}}{a^{2} q_{0}}+1}\right\} .
$$

With the help of (3.14), the above yields the differential inequality

$$
V^{\prime}(t)+\mu V(t)<0 \quad \forall t \in R^{+},
$$


where

$$
0<\mu=\frac{2 \lambda}{k}\left(1-\frac{\bar{k} \lambda}{a^{2} k p_{0}}\right) \frac{1}{1+\frac{\lambda}{k}\left(\frac{\sqrt{k}}{a}+\frac{2 \lambda}{a^{2}}+\frac{p_{1} \bar{k}}{a^{2} q_{0}}+\frac{p_{1}}{q_{0}}+1\right)} .
$$

Multiplying (3.20) by $e^{\mu t}$ and integrating over the time interval $[0, t]$, we get the estimate

$$
V(t)<e^{-\mu t} V(0) \quad \forall t \in R^{+} .
$$

Invoking the inequality (3.14) again in (3.22), we have

$$
E(t)<M e^{-\mu t} E(0) \quad \forall t \in R^{+},
$$

where

$$
M=\frac{1+\frac{\lambda}{k}\left(\frac{\sqrt{k}}{a}+\frac{2 \lambda}{a^{2}}+\frac{\bar{k}}{a^{2} q_{0}}+\frac{p_{1}}{q_{0}}+1\right)}{1-\frac{\lambda}{k}\left(\frac{\sqrt{k}}{a}+\frac{\bar{k}}{a^{2} q_{0}}+1\right)} .
$$

The finishes the proof of the theorem.

\section{REFERENCES}

[1] G.C. Gorain, Exponential eneragy decay estimates for the solutions of $n$-dimensional Kirchhoff type wave equation, Applied Mathematics and Computation 117, 235-242 (2006).

[2] M.A. Horn, Exact controllability and uniform stabilization of the Kirchhoff plate equation with boundary feedback acting via bending moments, J. Math. Anal. Appl. 167, 557-581 (1992).

[3] G. Kirchhoff, Vorlesungen übear Mathematische Physik, Mechanik(Teubner) 1977.

[4] J.Y. Park and S.H. Park, Decay rate estimates for wave equations of memory type with acoustic boundary conditions, Nonlinear Analysis : Theory, methods and Applications 74(3), 993-998 (2011).

[5] J.T. Beal and S.I. Rosencrans, Acoustic boundary conditions, Bull. Amer. Math. Soc. 80, 1276-1278 (1974).

[6] C.L. Frota and J.A. Goldstein, Some nonlinear wave equations with acoustic boundary conditions, J. Differ. Equ. 164, 92-109 (2000).

[7] H. Harrison, Plane and circular motion of a string, J. Acoust. Soc. Am. 20, 874-875 (1948).

[8] A.T. Cousin, C.L. Frota and N.A. Larkin, On a system of Klein-Gordon type equations with acoustic boundary conditions, J. Math. Anal. Appl. 293, 293-309 (2004).

[9] C.L. Frota and N.A. Larkin, Uniform stabilization for a hyperbolic equation with acoustic boundary conditions in simple connected domains, Progr. Nonlinear Differential Equations Appl. 66, 297-312 (2005).

[10] Y.H. Kang, M.J. Lee and I.H. Jeong, Stabilization of Kirchhoff type wave equation with locally distributed damping, Applied Mathematics Letters. 22(5), 719-722 (2009).

[11] J.Y. Park and J.A. Kim, Some nonlinear wave equations with nonlinear memory source term and acoustic boundary conditions, Numer. Funct. Anal. Optim. 27, 889-903 (2006).

[12] J.Y. Park and T.G. Ha, Well-posedness and uniform decay rates for the Klein-Gordon equation with damping term and acoustic boundary conditions, J. Math. Phys. 50 (2009) Article No. 013506; doi:10.1063/1.3040185

[13] A. Vicente, Wave equations with acoustic/memory boundary conditions, Bol. Soc. Parana. Mat. 27(3), 29-39 (2009). Springer-Verlag, New York, 1972. 